NASA Technical Memorandum 103646

AIAA-91-0284

\title{
Dropsize Correlation for Cryogenic Liquid-Jet Atomization
}

Richard D. Ingebo

Lewis Research Center

Cleveland, Ohio

Prepared for the

29th Aerospace Sciences Meeting

sponsored by the American Institute of Aeronautics and Astronautics

Reno, Nevada, July 7-10, 1991

\section{NMSA}





\author{
Robert $D$. Ingebo \\ National Aeronautics and Space tdministration \\ Lew is Research Center \\ Cleveland, Ohio $\$ 4135$
}

\section{Abstract}

Momentum transfer from high velocity nitrogen gasflow to liquid-nitrogen jets was investigated. $A$ correlation of aerodynamic and liquid-surface forces with characteristic drop diameter was obtained for cryogenic liquid-jet breakup in Mach 1 gasflow. Nitrogen gas mass-flux was varied from 5 to $17 \mathrm{~g} / \mathrm{cm}^{2}$ sec by using three differently sized two-fluid fuel atomizers with nozzle diameters varying from 0.30 to $0.56 \mathrm{~cm}$. A correlation was derived by using acoustic gas velocity, $V_{C}$, as a basic parameter in defining and evaluating the dimensionless product of the Weber and Reynolds numbers as follows: WeRe $=\rho_{g} \mathrm{D}_{0}^{2} v_{c}^{3} / \mu_{1}$ o where $\rho g$ is gas density, $D_{0}$ is liquid-flow orif ice diameter, $V_{C}$ is the acoustic velocity of the gas, $\rho$ is the liquid viscosity and $\sigma$ is the liquid surface tension. By using the definition of WeRe given above. it was found that the ratio of orifice diameter to Sauter mean drop diameter, $D_{0} / D_{32}$, could be correlated with the dimensionless ratio WeRe and the gas to liquid density ratio. $\rho_{g} / \rho_{l}$. as follows:

$$
D_{0} / D_{32}=8.1\left(\text { WeRe } \rho_{g} / \rho_{1}\right)^{0.44}
$$

From this expression, it is evident that

$D_{32} \sim V_{C}^{-1.33}$ which agrees very well with atomiza$t$ ion theory for the case of acceleration-wave breakup of liquid jets in high velocity gasflow.

\section{Nomenc la ture}

$A_{0}$ atomizer orifice area, $\mathrm{cm}^{2}$

$\mathrm{D}_{i} \quad$ diameter of $i^{\text {th }} \mathrm{drop}, \mathrm{cm}$

$D_{\mathrm{V} .5}$ volume median drop diameter for entire spray, cm

Dy.75 drop diameter such that 75 percent of the total liquid volume is in drops of smaller diameter

$D_{31}$ volume-linear mean drop diameter. $\left[\sum n_{i} D_{i}^{3} / \sum n_{i} D_{i}\right]^{0.5}$, cm

$\mathrm{D}_{32}$ Sauter mean drop diameter, $\sum n_{i} b_{i}^{3} / \sum n_{i} D_{i}^{2}$, cm

n number of droplets

V fluid velocity, cm/sec

w weight flow of fluid, g/sec

$\overline{\mathbf{x}} \quad$ axial downstream spray sampling distance. $\mathrm{cm}$

p density of fluid, $g / \mathrm{cm}^{3}$

Copyright (c) 1991 by the American Institute of Aeronautics and Astronatics. Inc. No copyright is asseried in the United States under Title 17. U.S. Code. The U.S. Government has a royalty-free license to exercise all rights under the copyright claimed herein for Governmental purposes.

All other rights are reserved by the copyright owner.
Subscripts:

c acoustic
g nitrogen gas

l liquid

\section{Introduction}

Cryogenic liquid-jets injected with assist gasflow into rocket combustors are quickly disintegrated into small-droplet sprays. In order to calculate vaporization or burning rates, it is necessary to characterize the spray in terms of drop size distribution and mean drop sizes such as Sauter mean or volume median diameters. Once characteristic drop sizes are known, mathematica! expressions can be derived to adequately describe a two-fluid atomization process in which various liquid-propellants and atomizing-gas combinations are used to enhance spray combustion and yield high combustor performance over wide ranges of operating conditions.

Two-phase flow and the transfer of momentum from highly turbulent Mach 1 gasflow to the surface of liquid-nitrogen jets was experimentally investigated. Three different pneumatic two-fluid nozzles were used to produce clouds of liquidnitrogen drops and tests were conducted primarily in the aerodynamic stripping regime of disintegrating liquid jets. A scattered-light scanning instrument developed at NASA Lewis Research Center was used to measure characteristic drop diameters of the cryogenic-liquid sprays. By correcting for gas-turbulence and thermal-gradient effects on measurements, reproducible data could be obtained with the scattered-light scanner. To avoid the loss of small liquid-nitrogen drops due to their high vaporization rate, measurements were taken close to the nozzle orifice.

Prior to the present study, an investigation of water sprays was made with two-fluid fuel nozzles and good agreement of experimental results with atomization theory was obtained as discussed in Ref. 1. It was found that the Sauter mean diameter, $D_{32}$, could be correlated with nitrogen gas flowrate. $w_{n}$. raised to the -1.33 power, which agrees well with theoretical expressions for liquid-jet breakup in high velocity gasflow. As a continuation of this study, the present investigation was initiated to extend experimental conditions to include variations in fuel nozzle orifice-area with the use of cryogenic liquids.

The fluid property that has the greatest effect on drop size of sprays produced with twofluid nozzles is the gas velocity relative to that of the liquid jet. Although numerous investigators have studied this phenomena as discussed in Refs. 2 to 6 , the effect of gas mass-flux on the drop size of cryogenic sprays has not yet been established in the spray literature. Therefore, the objective of the present study was to determine the effect of 
the atomizing gas mass-flux on liquid-nitrogen spray characteristics.

A correlation of aerodynamic and liquidsurface forces with characteristic drop diameter was obtained for liquid-nitrogen jets breaking up in Mach 1 gasflow. The atomizing nitrogen gas mass-flux was varied from 5 to $15 \mathrm{~g} / \mathrm{cm}^{2}-\mathrm{sec}$ by using three differently sized two-fluid atomizers with nozzle orifice areas varying from 0.112 to $0.345 \mathrm{~cm}^{2}$. The correlation was derived by using acoustic gas velocity, $V_{C}$, as a basic parameter in defining and evaluating the dimensionless product of the Weber and Reynolds numbers, WeRe.

\section{Apparatus and Procedure}

A pneumatic two-fluid atomizer was mounted in the test section as shown in Fig, 1 and a detailed diagram of the atomizer is shown in Fig. 2. The optical system of the scattered-light scanner shown in Fig. 1 measured scattered-light as a function of scattering angle by repeatedly sweeping a variable-length slit in the focal plane of the collecting lens. The data obtained is scattered-light energy as a function of scattering angle relative to the laser-beam axis. This method of particle size measurement is similar to that given in Ref. 7 and it is described in detail in Ref. 8 .

Characteristic drop sizes were obtained by locating four points on a plot of scattered-light energy which is normalized to the maximum energy and plotted against scattering angle. As illustrated in Fig. 3, the points gave Sauter mean, volume median, volume $l i n e a r$ and 75 -percent volume drop diameters. In Ref. 8, it is demonstrated that the four characteristic drop diameters and the dispersion of drop sizes are substantially independent of particle size distribution function. For a typical measurement, the scan is repeated 60 times per second to average out any temporal variations in the energy curve.

By measuring characteristic drop diameters for the entice spray cross section, spray pattern affects were minimized and reproducibility of characteristic drop size measurements was within \pm 5 percent. The instrument was calibrated with five sets of monosized polystyrene spheres having diameters of $8,12,25,50$, and $100 \mu \mathrm{m}$. Since the sprays were sampled very close to the atomizer orifice, they contained a relatively high number density of very small drops. As a result, lightscattering measurements required correction for multiple scattering as described in Refs. 8 and 9 for the case of high number-density sprays. Drop size measurements were also corrected as described in Ref. 8 to include Mie scattering theory when very small characteristic drop diameters, i.e., $<10 \mu \mathrm{m}$, were measured.

When characteristic drop sizes of liquidnitrogen sprays were first measured, it was found that good reproducible measurements were difficult to obtain with cryogenic sprays. This was attributed to excessively high temperature gradients in the gas-phase due to the low temperature of the liquid-nitrogen droplets, $77 \mathrm{~K}$. Density variations caused by high temperature gradients deflected the laser beam and caused a spike to appear on the light energy curve as shown in Fig. 4(a). To reduce the amplitude of the large spike, the laser beam was moved $0.6 \mathrm{~mm}$ from the path of the rotating variable-length slit shown in Fig. 1. This adjustment, described in detail in Ref. 10. causes a distortion of the true scattered-light wave shape at a small scatter angle, which is corrected by instrument calibration. The spike was reduced as shown in Fig. $+(b)$ by computations employing the equations given in Ref. 8. As a result, it was possible to obtain good reproducible drop size data for cryogenic sprays with the scattered-light scanning instrument.

As shown in Fig, 1, air supplied at ambient temperature, $293 \mathrm{~K}$, passed through the $15.24 \mathrm{~cm}$ inside diameter test section which exhausted to the atmosphere. The test section was $1 \mathrm{~m}$ in length and a $5.08 \mathrm{~cm}$ diameter orifice was used to measure airflow rate in the test section. A flow of dry air at a velocity of $5 \mathrm{~m} / \mathrm{sec}$ was maintained in the test section to aid in transporting small droplets through the laser beam. This prevented ambient humid air from contacting the spray and thereby avoided the formation of ice particles in the laser beam near the atomizer office.

The atomizer shown in Fig. 2 was mounted at the center line of the test duct and operated over pressure ranges of 0.2 to $1.0 \mathrm{MPa}$ for both liquid nitrogen and the atomizing gas. Liquid nitrogen sprays were injected downstream into the airflow just upstream of the duct exit. The sprays were sampled at a distance of $1.3 \mathrm{~cm}$ downstream of the atomizer orifice with a 4.4 by $1.9 \mathrm{~cm}$ rectangular laser beam produced by the aperture shown in Fig. 2. A diagram of the pneumatic two-fluid nozzles used in this study is shown in Fig. 3. Orifice areas for the three nozzles was varied from 0.112 to $0.345 \mathrm{~cm}^{2}$, as shown in Table 1 .

Liquid nitrogen at a temperature of $77 \mathrm{~K}$ measured with an I.C. thermocouple was axially injected into the airstream at $27.5 \mathrm{~g} / \mathrm{sec}$ as indicated by a turbine flow meter. Nitrogen gas was then turned on to atomize the liquid-nitrogen jet and weight flow rate was measured with a $0.51 \mathrm{~cm}$ diameter sharp-edge orifice. After the air, nitrogen gas and liquid-nitrogen flow rates were set. characteristic drop diameters were determined from measurements made with the scattered-light scanner.

\section{Experimental Results}

Pneumatic two-fluid nozzles $M-1, \# 4$ and $Q_{\text {rn }}$ were tested with liquid, $\mathrm{LN}_{2}$, and gaseous, $\mathrm{GN}_{2}$, nitrogen to determine the effect of $\mathrm{GN}_{2}$ flowrate on the characteristic drop size, $D_{32}$, of cryogenic sprays. Dimensions for the three nozzles are given in Table 1. The effect of $\mathrm{GN}_{2}$ mass-flux on $\mathrm{D}_{32}$ was also investigated, and a correlating expression was obtained for the three nozzles.

\section{Ef fect of $\mathrm{CN}_{2}$ Flowrate on $\mathrm{D}_{32}$}

Samples of the entire spray cross section were taken with the scattered-light scanner at a distance of $\bar{x}=1.3 \mathrm{~cm}$ and $a \mathrm{LN}_{2}$ flowrate of $27.5 \mathrm{~g} / \mathrm{sec}$. A characteristic drop diameter, $D_{32}$ was measured for each $\mathrm{LN}_{2}$ spray produced by the three nozzles and plotted against $\mathrm{Gi}_{2}$ flowrate. $w_{g}$. From this plot, the following relationship wâs obtained:

$$
\mathrm{D}_{32}^{-1} \sim w_{8}^{1.33}
$$


This expression agrees very well with that predicted by atomization theory given in Ref. 11 for liquid-jets disintegrating in high velocity gas streams, i.e., in the regime of aerodynamicstripping.

Effect of $\mathrm{GN}_{2}$ Mass-Flux on $\mathrm{D}_{32}$

Reciprocal SMD, $D_{32}^{-1}$ is plotted against $\mathrm{GN}_{2}$ flowrate per unit area as shown in Fig. 5. From this plot, the following relationship is obtained for the three nozzles:

$$
D_{32}^{-1}=k\left(w_{g} / A_{0}\right)^{1.33}
$$

where $k$ is a correlation coef $f$ icient that is a function of the physical properties of the two fluids. Values of $W_{g} / A_{O}$ were measured experimentally and equated to $\mathrm{GN}_{2}$ mass-flux since. $\mathrm{N}_{\mathrm{g}} / \mathrm{A}_{\mathrm{O}}=$ $\rho_{\mathrm{g}} \mathrm{V}_{\mathrm{g}}$. Therefore, Eq, 2 may be rewritten as:

$$
D_{32}^{-1}=k\left(p_{g}\right)^{1.33}
$$

The value of the correlation coef $f$ icient $k$ is 58 for the 3 nozzles. Some of the scatter of the data in Fig. 5 is attributed to the fact that $L_{2}$ jet diameter, $D_{0}$, varied from 0.295 to $0.564 \mathrm{~cm}$ for the three nozzles as indicated in Table 1 Values of $D_{32}^{-1}$ are somewhat greater for nozzle $M-1$, which has a smaller value of $D_{0}$, than that of nozzle $Q_{m}$. This is in agreement with Ref. 12 where it was found that $D_{32}^{-1} \sim D_{0}^{-0.2}$ in the study of water sprays produced with pneumatic two-fluid nozzles.

Correlation of Normalized Reciprocal SMD, $D_{32}^{-1}$ with Dimensionless Groups

Reciprocal SMD, $D_{32}^{-1}$, was normalized with respect to $\mathrm{LN}_{2}$ orifice diameter, $D_{0}$, and plotted against the product of three dimensionless groups, i.e., the ratio of gas to liquid density, $p g / p l$, the Weber number, We and Reynolds number, Re as shown in Fig. 6. From this plot, the following expression is obtained:

$$
\mathrm{D}_{\mathrm{o}} / \mathrm{D}_{32}=8.1\left(\text { WeRe } \rho_{\mathrm{g}} / \rho_{1}\right)^{0.44}
$$

where WeRe $\rho_{g} / \rho_{1}=D_{o}^{2}\left(\rho_{g} V_{c}\right)^{3} / \rho_{1} \mu_{1} \sigma, V_{c}$ is the acoustic velocity of the gas and 8.1 is a correlating constant for the three atomizers tested over the range of conditions used in this study.

\section{Summary of Results}

It was found that the fluid mechanics of cryogenic liquid-jet breakup in high velocity gasflow is quite difficult to study. This is primarily due to the fact that surface temperatures of liquidnitrogen jets used in the present study were always near their boiling point of approximately $77 \mathrm{~K}$. Since the atomizing gas was at room temperature, approximately $293 \mathrm{~K}$, this created large temperature gradients that deflected the laser beam, i.e.. caused beam steering to occur, when characteristic drop diameters were measured with the scattered-light scanner. This problem was minimized in the present investigation by moving the laser beam away from the slit in the scatteredlight scanner optical system.

Characteristic drop diameters of cryogenic liquid-nitrogen sprays were measured with a scattered-light scanning instrument at a distance of $1.3 \mathrm{~cm}$ downstream of the fuel nozzle orifices of three different pneumatic two-fluid atomizers. Values of $D_{32}^{-1}$ were correlated with nitrogen gas flowrate raised to the 1.33 power. This exponent of 1.33 is the same as that derived theoretically in Ref. 11 for liquid-jet breakup in the aerodynamic-stripping regime of atomization. The fact that experimental results agreed well with atomization theory was attributed to a negligible loss of small droplets due to vaporization and dispersion affects. As a result the following expression was derived for liquid-nitrogen sprays: $D_{0} / D_{32}=k$ (WeRe $\left.\rho_{g} / \rho_{1}\right)^{0.44}$, which agrees well with atomization theory for liquid-jet breakup in highvelocity gasflow, Ref. 11 .

In this study, results were obtained for liquid nitrogen jets breaking up at temperatures near the boiling point of liquid nitrogen. whereas previous water spray results, such as those given in Ref. 1, were obtained for water jets injected at room temperature conditions. As a result, these data cannot be compared directly. Data for water jets breaking up at temperatures near the boiling point of water are needed for comparison with cryogenic liquid-jet breakup data.

\section{References}

1. Ingebo, R.D., "Experimental and Theoretical Effects of Vitrogen Cas Flowrate on Liquid-Jet Atomization," Lournal of Propulsion and Power. Vol. 4, No. 6, Nov. -Dec. 1988, pp. 406-t11.

2. Kim, K.Y., and Marshall, W.R., Jr., Drop Size Distributions from Pneunatic Atomizers," Journal, Vol. 17, No. 3, May 1971, pp. $57 \overline{5-584}$.

3. Lorenzet to, G.E., and Lefebure, A.H., "Measurements of Drop Size on a Plain-Jet Airblast Atomizer," AlAA Journal, Vol. 15, Vo. 7, July 1977. pp. 1006-1010.

4. Nukiyama, S., and Tanasawa, Y., "Experiments on the Atomization of Liquids by Means of a Air Stream, Parts III-IV," Transactions of the Society of Mechanical Engineers, Japan. Vol. 5 , No. 18, Feb. 1939, pp. 63-75.

5. Weiss, M.A., and Worsham, C.H., "Atomization in High Velocity Airstreams," American Rocket Society Journal, Vol. 29, No. 4, Apr. 1959, pp. 252-259. 
6. Wolf, H.E., and Andersen, W.H., "Aerodynamic Break-up of Liquid Drops," Proceedings of the 5 th International Shock Tube Symposium. Z.I. Slawasky, J.F. Moulton, Ir., and W.S. Filler. Eds., Naval 0rdinance Lab., White Oak, MD, 1965, pp. 1145-1169. (Avail. VTIS, AD-638011).

7. J. Swi thenbank, J.M. Beer, D.S. Taylor, D. Abbot, and G.C. McCreath, "A Laser Diagnostic Technique for the Measurement of Droplet and Particle Size Distribution," Experimental Diagnostics in Gas Phase Combustion Systems B.T. Zinn, and C.T. Bowman, Ed. Progress in Astronautics and Aeronautics, Vol. 53, AlAA, New York, 1977, pp. $\$ 21-447$.

8. D.R. Buchele, "Particle Sizing by Weighted Measurements of Scattered Light," NASA

TM-100968, 1988.
9. P.G. Felton, A.A. Hamidi, and A.K. Aigal, "Measurement of Drop Size Distribution in Dense Sprays by Laser Diffraction," ICLASS-85: Proceedings of the Third International Conference on Liquid Atomization and Spray Systems . Vol. 2, P. Eisenklam, and A. Yule, Eds.. Inst $i$ tute of Energy, London, 1985, pp. [VA/4/1$[\mathrm{VA} / 4 / 11$.

10. D.R. Buchele, "Particle Sizing by Measurement of Forward-Scattered Light at Two Angles. NASA TP-2156, 1983

11. M. Adelberg. "Mean Drop Size Resulting from the Injection of a Liquid Jet Into a HighSpeed Gas Stream." AIAA Journal. Vol. 6, June 1968. pp. 1143-1147.

TABLE 1. - PNELMATIC TWO-FLLID FUEL YOZZLE

CHARACTERISTICS

\begin{tabular}{|c|c|c|c|}
\hline Fuel nozzle & $\begin{array}{l}D_{0} \\
\text { cm }\end{array}$ & $\begin{array}{l}\mathrm{D}_{\mathrm{n}} \\
\mathrm{cm}\end{array}$ & ${ }_{\mathrm{Cm}_{2}}^{\mathrm{C}^{2}}$ \\
\hline $\begin{array}{l}M-1 \\
\# 4 \\
Q_{m}\end{array}$ & $\begin{array}{r}0.295 \\
.375 \\
.564\end{array}$ & $\begin{array}{r}0.360 \\
.580 \\
.663\end{array}$ & $\begin{array}{r}0.112 \\
.264 \\
.345\end{array}$ \\
\hline
\end{tabular}

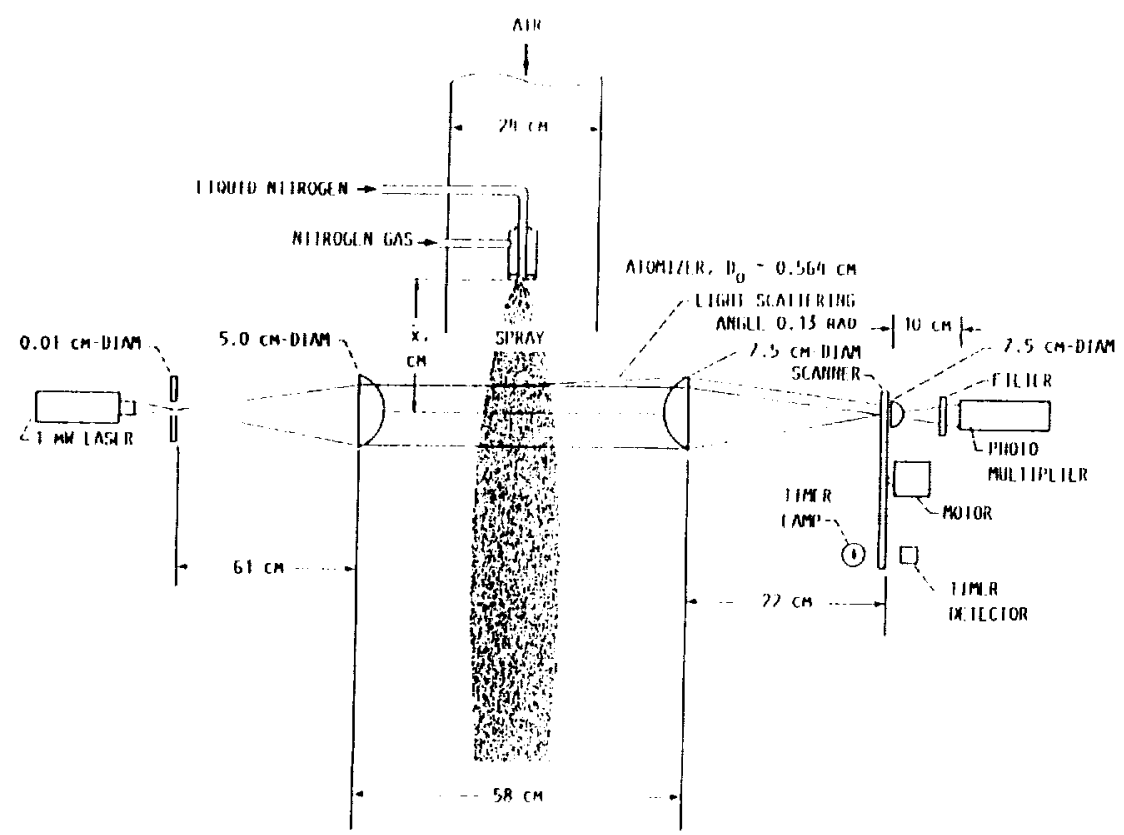

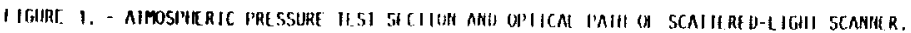

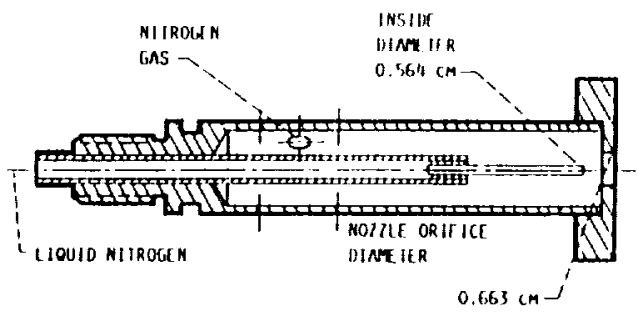

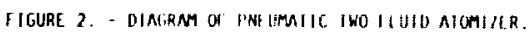

